\title{
A minimally invasive lateral approach with CT navigation for open biopsy and diagnosis of Nocardia nova L4-5 discitis osteomyelitis: illustrative case
}

\author{
Bryan Zheng, BS, Hael Abdulrazeq, MD, Owen P. Leary, BS, Ziya L. Gokaslan, MD, Adetokunbo A. Oyelese, MD, PhD, Jared S. Fridley, MD, \\ and Joaquin Q. Camara-Quintana, MD
}

Department of Neurosurgery, Warren Alpert Medical School of Brown University, Providence, Rhode Island

\begin{abstract}
BACKGROUND Lumbar spine osteomyelitis can be refractory to conventional techniques for identifying a causal organism. In cases in which a protracted antibiotic regimen is indicated, obtaining a conclusive yield on biopsy is particularly important. Although lateral transpsoas approaches and intraoperative computed tomography (CT) navigation are well documented as techniques used for spinal arthrodesis, their utility in vertebral biopsy has yet to be reported in any capacity.

OBSERVATIONS In a 44-year-old male patient with a history of Nocardia bacteremia, CT-guided biopsy failed to confirm the microbiology of an L4-5 discitis osteomyelitis. The patient underwent a minimally invasive open biopsy in which a lateral approach with intraoperative guidance was used to access the infected disc space retroperitoneally. A thin film was obtained and cultured Nocardia nova, and the patient was treated accordingly with a long course of trimethoprim-sulfamethoxazole.
\end{abstract}

LESSONS The combination of a lateral transpsoas approach with intraoperative navigation is a valuable technique for obtaining positive yield in cases of discitis osteomyelitis of the lumbar spine refractory to CT-guided biopsy.

https://thejns.org/doi/abs/10.3171/CASE20164

KEYWORDS discitis osteomyelitis; image-guided biopsy; minimally invasive surgery; neuronavigation; Nocardia

Osteomyelitis of the lumbar vertebrae can be a cause of low back pain and most often requires antibiotics and conservative measures for pain control. However, late recognition and treatment can lead to hospital mortality rates ranging from $2 \%$ to $17 \% .{ }^{1-3}$ Vertebral osteomyelitis, also referred to as "discitis osteomyelitis" or "spondylodiscitis," is caused via hematogenous spread from a distant focus. ${ }^{4}$ Staphylococcus aureus is known to be the most common causal pathogen accounting for about half of nontuberculosis cases. ${ }^{5,6}$

Magnetic resonance imaging (MRI) is commonly accepted as the radiological modality of choice for diagnosis and characterizing the extent of involvement. 2,7,8 Computed tomography (CT) of the lumbar spine can be used to provide detailed visualization of bone involvement and is often used for guidance during biopsy. Blood cultures and bone biopsy have equal ability to isolate a causal organism, although blood cultures are obtained in almost all patients as a first diagnostic test owing to the relative ease and reduced risk of the procedure. ${ }^{9} \mathrm{CT}$ guided biopsy (CTGB) is usually performed when other studies lack the ability to yield a pathogen and there is a targetable lesion on MRI. However, even CTGB has been shown to offer modest yield, with the diagnostic power dependent on various clinical features. ${ }^{10-12}$ As an alternative, minimally invasive options for open biopsy offer novel ways to obtain microbiological yield from suspected vertebral infections in patients who had prior negative CTGBs. ${ }^{13,14}$

Although the role of intraoperative navigation for biopsy is poorly defined, it can be used as a powerful adjunct to traditional mini-open or tubular minimally invasive surgery (MIS). ${ }^{15}$ We present a case report of an MIS retroperitoneal transpsoas approach in the lateral decubitus position with the use of neuronavigation for open biopsy of an L4-5 vertebral osteomyelitis/discitis infection. The patient was a 44-year-old male with prior pulmonary nocardiosis and initial nondiagnostic CTGB. There have been prior reports of Nocardia spp. as causes of vertebral osteomyelitis but none using our technique. ${ }^{16,17}$ Of note, the Nocardia spp. are frequently intractable to positive biopsy by traditional means. ${ }^{18,19}$ In the literature, both CTGB and the few alternative MIS

ABBREVIATIONS CT = computed tomography; CTGB = computed tomography-guided biopsy; IV = intravenous; MIS = minimally invasive surgery; MRI = magnetic resonance imaging; PICC = peripherally inserted central catheter.

INCLUDE WHEN CITING Published March 1, 2021; DOI: 10.3171/CASE20164.

SUBMITTED December 22, 2020. ACCEPTED January 6, 2021.

(C) 2021 The authors, CC BY-NC-ND 4.0 (http://creativecommons.org/licenses/by-nc-nd/4.0/), 
biopsy techniques have been attempted through a posterior or posterolateral approach. We believe that this is the first reported lateral MIS approach to address this scenario, utilizing both navigation and a tubular transpsoas technique to obtain a positive culture yield.

\section{Illustrative Case}

A 44-year-old male with a history of prior Nocardia bacteremia was admitted from the Emergency Department with the chief complaint of worsening lower back pain and a recent hospitalization for L4-5 osteodiscitis with paravertebral phlegmon. Nine months earlier the patient had been treated with a course of trimethoprim-sulfamethoxazole and imipenem at a separate facility for Nocardia bacteremia. A CTGB performed approximately 2 weeks prior to the index admission was nondiagnostic with no organisms and few neutrophils. Blood cultures were also found to be negative except for one set with coagulasenegative gram-positive cocci suggestive of Staphylococcus that was believed to be a likely contaminant. Nevertheless, the patient was treated with vancomycin and piperacillin-tazobactam. He was discharged and planned to follow up with the Infectious Disease Department.

Upon readmission, the patient was experiencing progressively worse back pain, as well as left hip pain, despite taking pain medications and his new antibiotics. His neurological examination revealed normal motor function and sensation in the lower extremities. Initial laboratory results were all normal, including a set of negative blood cultures. An MRI study of the lumbar spine showed stable discitis or osteomyelitis from the MRI 2 weeks prior (Fig. 1). The infectious disease team consulted interventional radiology for another CTGB, but the patient was deemed a poor candidate given the prior negative result. We were eventually consulted and asked if any open approach could be undertaken to help obtain a positive yield. The infectious disease team wanted confirmation of presumed recurrent Nocardia infection before prescribing an additional trimethoprim-sulfamethoxazole regimen, which would require longer than 9 months of treatment and a more deleterious side-effect profile than alternatives for other diagnoses.
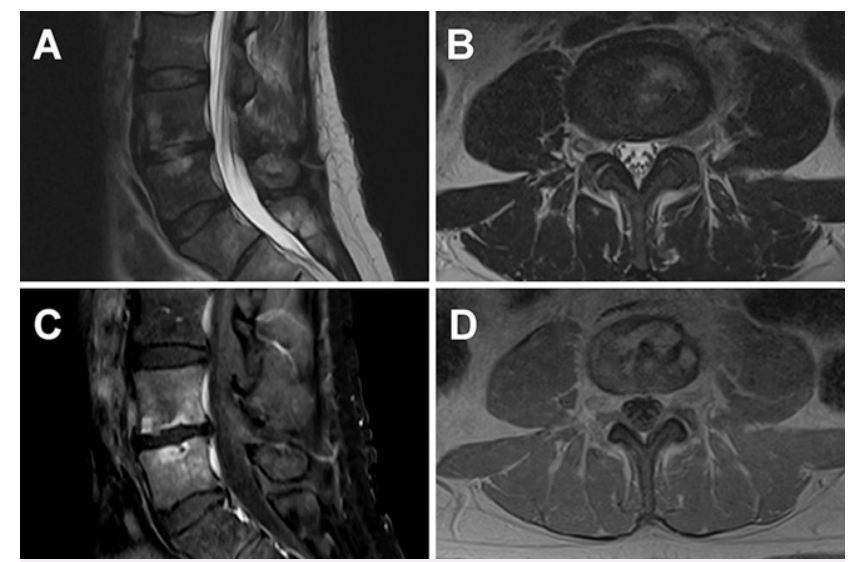

FIG. 1. MRI sequences of known L4-5 discitis osteomyelitis. A and B: T2 hyperintensity of the $L 4$ and $L 5$ vertebral bodies from sagittal and axial views were overall stable compared with MRI from earlier in the initial hospital course. C: Postgadolinium T1-weighted sagittal view demonstrated adjacent involvement of the inferior $L 4$ and superior L5 endplates. D: Axial slice of the inferior L4 endplate demonstrating contrast enhancement.
Given the location of the L4-5 osteomyelitis/discitis involving the outer lateral aspect of the adjacent vertebral body, we believed the lesion could be amenable to open biopsy via a lateral retroperitoneal transpsoas approach with real-time navigation. This approach would enable direct visual access to the lesion instead of a "blind" approach reliant on navigation alone or imaging from a posterior approach.

\section{Procedure Description}

The patient was brought into the operating room and intubated, then secured in the right lateral decubitus position. Fluoroscopy was brought into the room, and the L4-5 disc space was localized, as were the anterior and posterior margins of the L4-5 vertebral bodies. A $3-\mathrm{cm}$ incision was marked spanning the disc space level. The site was prepped in a sterile fashion. An iliac reference array was mounted to the left iliac crest, and an intraoperative CT scan was obtained (Fig. 2, AIRO $A G$, BrainLAB). A retroperitoneal transpsoas approach was performed, and the disc space was serially dilated with electrophysiological monitoring. Retractors (NuVasive) were placed, expanded, and electrically monitored for lumbar plexus irritation. Once expanded, the disc space and lesion were directly visualized and the level confirmed with navigation. At the inferior aspect of the $L 4$ vertebral body was a "punched-out" lytic lesion with a thin yellow film. We verified that the visualized lesion correlated to the intraoperative CT that was being used for navigation in real time (Fig. $2 \mathrm{C}$ and D). Of note, no pus was encountered in the psoas muscle, vertebral body, or disc space. The thin film in the punched-out lytic part of the vertebral body was curetted off and sent to microbiology. Retractors used to expand the psoas muscle were withdrawn, and the incision was closed in layers. Total retraction time was only 12 minutes, and there were no immediate complications or changes in neuromonitoring throughout the procedure. The patient woke up at his neurological baseline, notably without left thigh numbness or other evidence of postoperative plexopathy.

\section{Surgical Pathology}

The patient was discharged 3 days after the open biopsy with some mild incisional pain and good leg strength bilaterally. Antibiotics were withheld pending surgical pathology results, which returned as Nocardia spp 1 day after the patient's discharge. Isolate was also sent to the Mycobacteria/Nocardia Laboratory (University of Texas Health Center at Tyler) for further confirmation and matched $99.80 \%$ to Nocardia nova strains by $16 \mathrm{~S}$ rRNA gene sequencing.

The Infectious Disease Department notified the patient and requested he return to the inpatient service for a peripherally inserted central catheter (PICC) line and the recommended antibiotics. He began a dual course of intravenous (IV) trimethoprim-sulfamethoxazole and imipenem/cilastatin administered every 6 hours. After 6 weeks, the patient's PICC line was removed and he was transitioned to oral trimethoprim-sulfamethoxazole (480 mg, twice daily) for suppressive therapy with a plan to continue this for at least 1 year depending on his toleration. Upon the most recent follow-up, he reported significant improvement in his low back pain.

\section{Discussion \\ Observations}

A minimally invasive lateral retroperitoneal transpsoas approach to open biopsy using intraoperative CT for direct navigation successfully identified $N$. nova in a case of CTGB-refractory L4-5 discitis osteomyelitis. Our technique shows the success of this approach and also demonstrates that it should be a consideration for cases yielding no 

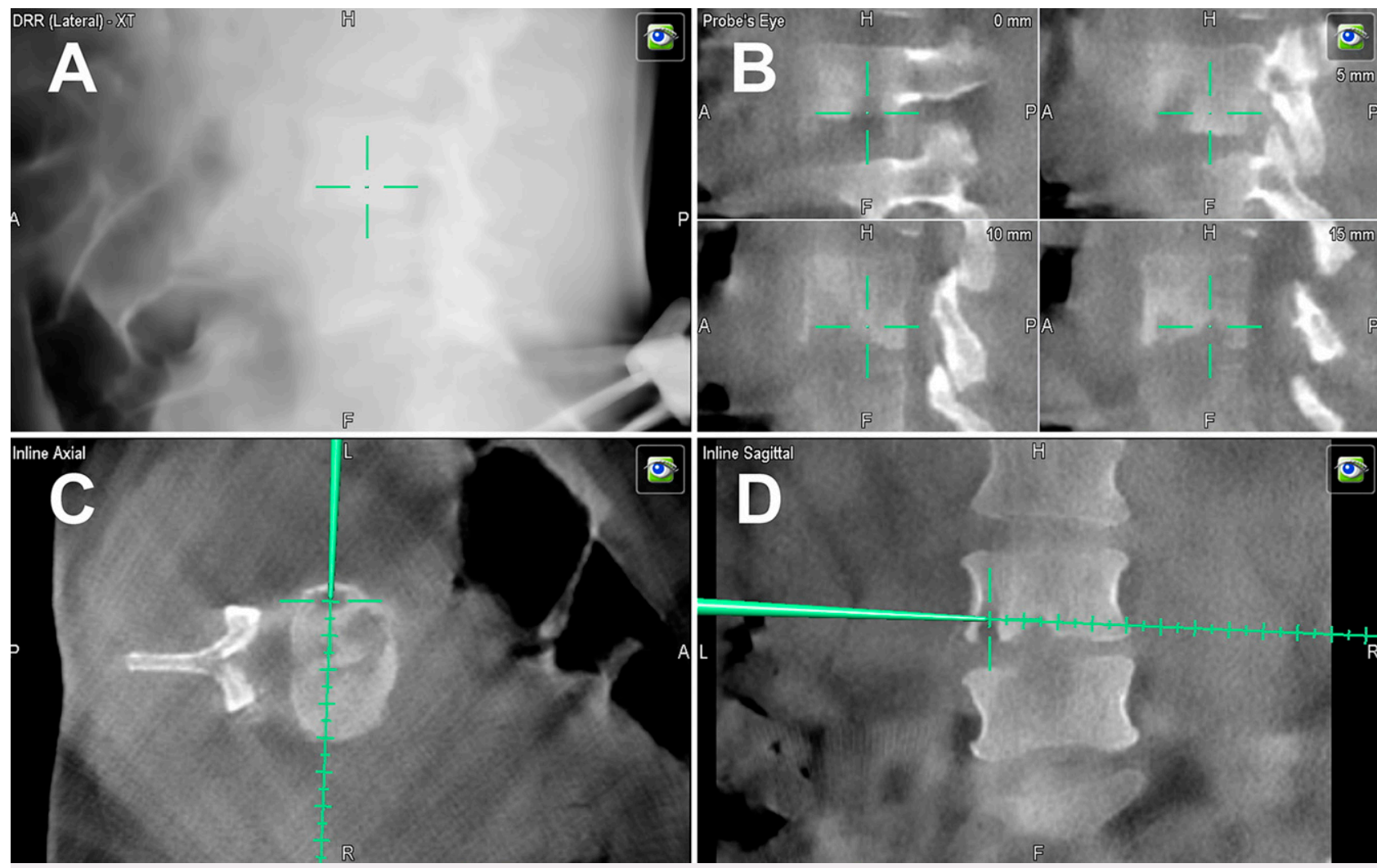

FIG. 2. Intraoperative image workflow used for direct guidance during lateral biopsy. A and B: After placement of our iliac pin for registration and obtaining our intraoperative CT (AIRO, BrainLAB), offset was used to ensure our incision correlated with our fluoroscopy to target the L4-5 disc space. C and D: After performing our retroperitoneal transpsoas approach with serial dilation of the disc space, our navigation wand showed our location and target of the lytic lesion.

result through CTGB or even as a first approach for diagnostic biopsy when difficult-to-biopsy pathogens are suspected.

This patient's lumbar spine infection was also caused by Nocardia, a genus known to be difficult to culture. In this case, an MIS lateral retroperitoneal transpsoas approach with navigation proved to be a viable option after failed CTGB. Our approach allowed for successful diagnosis and confirmed the need for treatment with specific antibiotics, helping both the infectious disease team and, more importantly, our patient.

\section{Lessons}

Although MIS of the spine encompasses various types of procedures, a common goal is to preserve the surrounding tissue and musculature in order to decrease the morbidity associated with open approaches, such as blood loss, and to facilitate improved functional recovery. ${ }^{20}$ Moreover, MIS avoids muscular and ligamentous disruption, which may lead to spinal instability. ${ }^{21}$ Originally described by Ozgur et al., the lateral transpsoas approach has become a proven and popular method for gaining access to the lumbar spine, particularly for indirect decompression, correction of deformity, and lumbar arthrodesis. ${ }^{22}$ This approach is increasingly preferred for access to intervertebral discs due to the minimization of blood loss, vascular injury, dural tear, postoperative pain, and hospital length of stay compared to those with anterior and posterior open techniques. ${ }^{23-26}$ This approach does increase the procedural risk of transient and permanent lumbar plexopathies, which can induce various lower extremity symptoms, including anterior thigh/groin pain, numbness, and weakness. ${ }^{27} \mathrm{Re}-$ cently, the MIS lateral retroperitoneal transpsoas approach has become a mainstay of spine surgery; however, it has rarely been used for diagnosing or treating spinal infections.

Real-time image guidance has become a powerful adjunct to MIS for spinal procedures. Although intraoperative fluoroscopy is traditionally used to visualize target anatomy, intraoperative CT navigation has been demonstrated across multiple studies and meta-analyses to provide greater surgical precision, enhanced orientation to patient 3-dimensional anatomy, and decreased intraoperative complication rates. ${ }^{28-30}$ Additionally, the use of intraoperative CT guidance has been shown to reduce radiation exposure to the surgeon and operating room staff compared with traditional fluoroscopy. With regard to limitations, the cost-effectiveness of image-guided surgery is not yet well described. ${ }^{31,32}$ There can also be a steep learning curve for surgeons learning to incorporate this technology into their MIS practice. However, there is no doubt that combining MIS and spinal navigation offers a powerful intraoperative toolkit for optimizing outcomes in some of our most challenging cases.

The applications of MIS approaches have been expanded to treat multiple spinal pathologies, including discitis or osteomyelitis, especially for patients with significant comorbidities that increase operative risk. ${ }^{33}$ For example, Yang et al. found favorable bacterial yields using percutaneous endoscopic biopsy, a technique similar to that used in this report in that it was originally used for treatment of other lumbar disc pathologies rather than biopsy. ${ }^{14}$ Still, there is a dearth of 
studies demonstrating the role and efficacy of the lateral retroperitoneal transpsoas approach for decompression and debridement of refractory discitis. ${ }^{34-36}$ Most reports of MIS techniques for spinal infections have focused on percutaneous CT-guided or fluoroscopy-guided needle biopsy, which can be more accessible and cost-effective but at times produce unreliable results for certain subsets of lesions. ${ }^{37}$

Reasons for the relatively low microbiological yield of CTGB in the setting of infection may include the lack of precise visualization of the target lesion, variable expertise of those performing the biopsy, indolent nature of the pathogen, and/or lesion size and composition. In most cases, if the traditional CTGB is nondiagnostic, no other biopsy options are typically offered, and the patient is treated empirically with broadspectrum antibiotics. Less targeted antibiotic treatment can be ineffective, have deleterious patient side effects, and lead to antibiotic resistance. Thus, MIS approaches have really expanded the armamentarium for lesions that are difficult to biopsy without direct visualization. In this case, we utilized a new approach: a direct lateral retroperitoneal transpsoas approach similar to that used in lateral lumbar interbody fusion, using intraoperative CT for direct real-time guidance. Our case shows that this is a viable option and should be considered in patients with discitis or osteomyelitis that has yielded inconclusive results from CTGB and lesions that are not targetable or difficult to target through traditional CTGB. Additionally, a transpsoas approach was chosen primarily because of its ease and familiarity to the institution but may not necessarily be optimal for all similar cases. Performing an MIS biopsy via a prepsoas approach could also be a reasonable option, particularly when navigation is used. Further studies that directly compare the reliability of these techniques with CTGB would be necessary to quantify benefits more clearly and to compare complication profiles.

Nocardia spp. are also notoriously slow growing and therefore difficult to culture. ${ }^{19}$ There is scant literature on Nocardia osteomyelitis in the vertebrae, with only one other case of $N$. nova spondylodiscitis reported by Hamdad et al. in 2007. ${ }^{16}$ This patient's infection differs in that there was no voluminous psoas abscess that could have easily drained purulence to yield the causative organism. Imipenem has been shown to be effective for the treatment of all $N$. nova strains, among other Nocardia spp..$^{38,39}$ Because imipenem/cilastatin often requires a protracted regimen with mandatory IV administration and significant adverse side effects, it was particularly important to confirm the suspected microbiology in this patient, who had a recent history of Nocardia bacteremia. ${ }^{40}$

\section{References}

1. Sobottke R, Seifert H, Fätkenheuer G, et al. Current diagnosis and treatment of spondylodiscitis. Dtsch Arztebl Int. 2008;105(10): 181-187.

2. Frangen TM, Kälicke T, Gottwald M, et al. Surgical management of spondylodiscitis. An analysis of 78 cases. Article in German. Unfallchirurg. 2006;109(9):743-753.

3. Schinkel C, Gottwald M, Andress HJ. Surgical treatment of spondylodiscitis. Surg Infect (LarChmt). 2003;4(4):387-391.

4. Katsevman GA, Emery E, France JC, Sedney CL. Secondary discitis masquerading as treatment failure of primary discitis: case report and review of the literature. Int J Spine Surg. 2019;13(2): 120-124.

5. Gouliouris T, Aliyu SH, Brown NM. Spondylodiscitis: update on diagnosis and management. J Antimicrob Chemother. 2010; 65(suppl 3):iii11-iii24.

6. Mylona E, Samarkos M, Kakalou E, et al. Pyogenic vertebral osteomyelitis: a systematic review of clinical characteristics. Semin Arthritis Rheum. 2009;39(1):10-17.
7. Maiuri F, laconetta G, Gallicchio B, et al. Spondylodiscitis. Clinical and magnetic resonance diagnosis. Spine (Phila Pa 1976). 1997; 22(15):1741-1746.

8. Glaser C, Matzko M, Reiser M. Chronic infections of the skeletal system. Their imaging diagnosis. Article in German. Radiologe. 2000;40(6):547-556.

9. Bhavan KP, Marschall J, Olsen MA, et al. The epidemiology of hematogenous vertebral osteomyelitis: a cohort study in a tertiary care hospital. BMC Infect Dis. 2010;10:158.

10. Ang MT, Wong GR, Wong DR, et al. Diagnostic yield of computed tomography-guided biopsy and aspiration for vertebral osteomyelitis. J Med Imaging Radiat Oncol. 2019;63(5):589-595.

11. Heyer CM, Brus LJ, Peters SA, Lemburg SP. Efficacy of CTguided biopsies of the spine in patients with spondylitis-an analysis of 164 procedures. Eur J Radiol. 2012;81(3):e244-e249.

12. Garg V, Kosmas C, Young PC, et al. Computed tomographyguided percutaneous biopsy for vertebral osteomyelitis: a department's experience. Neurosurg Focus. 2014;37(2):E10.

13. Turel MK, Kerolus M, Deutsch $H$. The role of minimally invasive spine surgery in the management of pyogenic spinal discitis. J Craniovertebr Junction Spine. 2017;8(1):39-43.

14. Yang SC, Fu TS, Chen LH, et al. Identifying pathogens of spondylodiscitis: percutaneous endoscopy or CT-guided biopsy. Clin Orthop Relat Res. 2008;466(12):3086-3092.

15. Holly LT, Foley KT. Intraoperative spinal navigation. Spine (Phila Pa 1976). 2003;28(15)(suppl):S54-S61.

16. Hamdad F, Vidal B, Douadi Y, et al. Nocardia nova as the causative agent in spondylodiscitis and psoas abscess. J Clin Microbiol. 2007;45(1):262-265.

17. Johnson P, Ammar H. Nocardia brasiliensis vertebral osteomyelitis and epidural abscess. BMJ Case Rep. 2013;2013: bcr2012008400.

18. McNeil MM, Brown JM. The medically important aerobic actinomycetes: epidemiology and microbiology. Clin Microbiol Rev. 1994;7(3):357-417.

19. Brown-Elliott BA, Brown JM, Conville PS, Wallace RJ Jr. Clinical and laboratory features of the Nocardia spp. based on current molecular taxonomy. Clin Microbiol Rev. 2006;19(2):259-282.

20. McAfee PC, Phillips FM, Andersson G, et al. Minimally invasive spine surgery. Spine (Phila Pa 1976). 2010;35(26 suppl): S271-S273.

21. Snyder LA, O'Toole J, Eichholz KM, et al. The technological development of minimally invasive spine surgery. BioMed Res Int. 2014;2014:293582.

22. Ozgur BM, Aryan HE, Pimenta L, Taylor WR. Extreme lateral interbody fusion (XLIF): a novel surgical technique for anterior lumbar interbody fusion. Spine J. 2006;6(4):435-443.

23. Rodgers WB, Gerber EJ, Patterson J. Intraoperative and early postoperative complications in extreme lateral interbody fusion: an analysis of 600 cases. Spine (Phila Pa 1976). 2011;36(1):26-32.

24. Lehmen JA, Gerber EJ. MIS lateral spine surgery: a systematic literature review of complications, outcomes, and economics. Eur Spine J. 2015;24(suppl 3):287-313

25. Lian X, Navarro-Ramirez R, Berlin C, et al. Total 3D Airo ${ }^{\circledR}$ navigation for minimally invasive transforaminal lumbar interbody fusion. BioMed Res Int. 2016;2016:5027340.

26. Salzmann SN, Shue J, Hughes AP. Lateral lumbar interbody fusion-outcomes and complications. Curr Rev Musculoskelet Med. 2017;10(4):539-546.

27. Sofianos DA, Briseño MR, Abrams J, Patel AA. Complications of the lateral transpsoas approach for lumbar interbody arthrodesis: a case series and literature review. Clin Orthop Relat Res. 2012; 470(6):1621-1632.

28. Tormenti MJ, Kostov DB, Gardner PA, et al. Intraoperative computed tomography image-guided navigation for posterior 
thoracolumbar spinal instrumentation in spinal deformity surgery. Neurosurg Focus. 2010;28(3):E11.

29. Tang J, Zhu Z, Sui T, et al. Position and complications of pedicle screw insertion with or without image-navigation techniques in the thoracolumbar spine: a meta-analysis of comparative studies. J Biomed Res. 2014;28(3):228-239.

30. Kosmopoulos V, Schizas C. Pedicle screw placement accuracy: a meta-analysis. Spine (Phila Pa 1976). 2007;32(3):E111-E120.

31. Al-Khouja L, Shweikeh F, Pashman R, et al Economics of image guidance and navigation in spine surgery. Surg Neurol Int. 2015; 6(suppl 10):S323-S326.

32. Lubelski D, Mihalovich KE, Skelly AC, et al. Is minimal access spine surgery more cost-effective than conventional spine surgery? Spine (Phila Pa 1976). 2014;39(suppl 1):S65-S74.

33. Karikari IO, Nimjee SM, Hardin CA, et al. Extreme lateral interbody fusion approach for isolated thoracic and thoracolumbar spine diseases: initial clinical experience and early outcomes. J Spinal Disord Tech. 2011;24(6):368-375.

34. Shepard M, Safain M, Burke SM, et al. Lateral retroperitoneal transpsoas approach to the lumbar spine for the treatment of spondylodiscitis. Minim Invasive Ther Allied Technol. 2014; 23(5):309-312.

35. Patel NB, Dodd ZH, Voorhies J, Horn EM. Minimally invasive lateral transpsoas approach for spinal discitis and osteomyelitis. J Clin Neurosci. 2015;22(11):1753-1757.

36. Ghobrial GM, Al-Saiegh F, Franco D, et al. Lateral lumbar retroperitoneal transpsoas approach in the setting of spondylodiscitis: a technical note. J Clin Neurosci. 2017;39:193-198.
37. Nam KH, Song GS, Han IH, et al. Diagnostic value of biopsy techniques in lumbar spondylodiscitis: percutaneous needle biopsy and open biopsy. Korean J Spine. 2011;8(4):267-271.

38. Lerner PI. Nocardiosis. Clin Infect Dis. 1996;22(6):891-905.

39. Saubolle MA, Sussland D. Nocardiosis: review of clinical and laboratory experience. J Clin Microbiol. 2003;41(10):4497-4501.

40. Wang C, Calandra GB, Aziz MA, Brown KR. Efficacy and safety of imipenem/cilastatin: a review of worldwide clinical experience. Rev Infect Dis. 1985;7(suppl 3):S528-S536.

\section{Disclosures}

Dr. Oyelese is a consultant for BrainLab and Depuy-Synthes.

\section{Author Contributions}

Conception and design: Abdulrazeq, Gokaslan, Fridley, CamaraQuintana. Acquisition of data: Abdulrazeq. Analysis and interpretation of data: Abdulrazeq. Drafting the article: Zheng, Abdulrazeq, Leary, Fridley. Critically revising the article: Zheng, Abdulrazeq, Leary, Oyelese, Fridley, Camara-Quintana. Reviewed submitted version of manuscript: Zheng, Abdulrazeq, Leary, Gokaslan, Camara-Quintana. Approved the final version of the manuscript on behalf of all authors: Zheng. Study supervision: Camara-Quintana.

\section{Correspondence}

Bryan Zheng: Warren Alpert Medical School of Brown University and Rhode Island Hospital, Providence, RI. bryan_zheng@brown.edu. 